\title{
Use of Ramelteon for the Treatment of Secondary REM Sleep Behavior Disorder
}

\author{
Takashi Nomura, Shinya Kawase, Yasuhiro Watanabe and Kenji Nakashima
}

\begin{abstract}
We used ramelteon to treat two patients with secondary REM sleep behavior disorder (RBD) complications along with neurodegenerative diseases including multiple system atrophy and Parkinson's disease. These two patients not only improved in terms of their clinical RBD symptoms but also exhibited a decrease in the proportion of REM sleep without atonia (from $8.5 \%$ to $3.5 \%$ and from $10.9 \%$ to $3.9 \%$ in the two patients). Although clonazepam is the standard first-line therapy for the treatment of RBD, ramelteon might be an effective treatment alternative in patients with RBD who cannot take clonazepam due to either ineffectiveness or adverse effects.
\end{abstract}

Key words: REM sleep behavior disorder, melatonin, REM sleep without atonia, Parkinson's disease, multiple system atrophy

(Intern Med 52: 2123-2126, 2013)

(DOI: 10.2169/internalmedicine.52.9179)

\section{Introduction}

REM sleep behavior disorder (RBD) is characterized by dream enactment with violent behavior, in addition to REM sleep without atonia (RWA) on polysomnography (PSG) (1). Clonazepam is the standard first-line treatment for RBD (2). Guidelines for the treatment of idiopathic RBD recommend a combination of clonazepam and melatonin as a level $\mathrm{B}$ treatment (3). However, in Japan, melatonin may not be used for treatment in clinical practice. Recently, the melatonin receptor agonist ramelteon was approved for the treatment of insomnia in Japan (4). We employed ramelteon therapy for two patients with secondary RBD, as the first patient continued to display RBD symptoms despite taking clonazepam and the second patient could not take clonazepam due to a progression of sleep apnea syndrome (SAS). We evaluated their clinical symptoms and polysomnographic findings related to RBD during the treatment period.

\section{Case Reports}

\section{Case 1}

In 2009, a 75-year-old, right-handed woman gradually began to display bradykinesia and a soft voice. At the same time, she frequently spoke during sleep. Subsequently, she complained about poor motor coordination such as difficulty in writing and dressing. In 2010, she was admitted to our hospital for an evaluation of her symptoms. Upon admission, she exhibited dysarthria, rigidity of the left limbs, bilateral hyperreflexia of the upper and lower limbs, a pathological reflex of the left upper limb and bilateral lower limbs, ataxia of trunk and limbs and urinary dysfunction. She exhibited dream-enactment behavior including arm movements and shouting while dreaming about being chased by strangers during sleep. She had severe orthostatic hypotension as evidenced by the drop in blood pressure (BP) from $135 / 76 \mathrm{mmHg}$ and a pulse of $81 \mathrm{bpm}$ to a $\mathrm{BP}$ of 98/ $64 \mathrm{mmHg}$ and a pulse of $82 \mathrm{bpm}$ on a head-up tilt table test. Cranial magnetic resonance imaging (MRI) revealed atrophic changes and a cross sign in the pons, atrophy and a high intensity in the T2-weighted images of the bilateral 
Table. Results of Polysomnographic Findigs

\begin{tabular}{lcccc}
\hline & \multicolumn{2}{c}{ case 1 } & \multicolumn{2}{c}{ case 2 } \\
\cline { 2 - 5 } & $\begin{array}{c}\text { before taking } \\
\text { ramelteon }\end{array}$ & $\begin{array}{c}\text { after taking } \\
\text { ramelteon }\end{array}$ & $\begin{array}{c}\text { before taking } \\
\text { ramelteon }\end{array}$ & $\begin{array}{c}\text { after taking } \\
\text { ramelteon }\end{array}$ \\
\hline Total sleep time (min) & 226.5 & 301 & 369.5 & 419 \\
Wake after sleep onset (\%) & 50.4 & 38.6 & 20.5 & 24.2 \\
Stage 1 (\%) & 6 & 11.9 & 22 & 14.8 \\
Stage 2 (\%) & 20.8 & 35.4 & 37.7 & 41.8 \\
Stage 3+4 (\%) & 10.4 & 2.2 & 0 & 0 \\
$\begin{array}{l}\text { Stage REM (\%) } \\
\text { Stage REM sleep without } \\
\text { atonia (\%) }\end{array}$ & 7.9 & 8.4 & 8.9 & 15.3 \\
$\begin{array}{l}\text { Periodic limb movement } \\
\text { index (/hour) }\end{array}$ & 8.5 & 3.5 & 10.9 & 3.9 \\
$\begin{array}{l}\text { Apnea hypopnea index } \\
\text { (/hour) }\end{array}$ & 6.6 & 91.9 & 23.4 & 0.1 \\
\hline
\end{tabular}

pedunculus cerebellaris medius (left predominant), atrophy of cerebellum and a linear high intensity of the T2-weighted images of the bilateral lateral portion of the putamen. Single photon emission computed tomography (SPECT) using technetium-99 m-ethyl cysteinate dimer revealed hypoperfusion of the cerebellar hemispheres (left predominant) and of the brain stem. ${ }^{123} \mathrm{I}$-metaiodobenzylguanidine (MIBG) myocardial scintigraphy indicated a normal heart to mediastinum $(\mathrm{H} / \mathrm{M})$ ratio on both the early and the delayed images (early image: 2.01, delayed image: 1.93). We also examined her PSG results. The sleep stages were scored according to the established criteria of Rechtschaffen and Kales (5). During REM sleep, the presence of submental phasic electromyogram (EMG) activity (3-second mini-epochs containing phasic twitches exceeding 4 times the background EMG activity) or submental tonic EMG activity (more than half of a 30 -second epoch in duration) was used to determine the stage of the RWA (6). The results of these PSG recordings are detailed in Table. The results of the PSG recordings revealed that she had RWA symptoms during $8.5 \%$ of her sleep time. She was diagnosed to have multiple system atrophy (MSA) per the established diagnostic criteria (7). Moreover, she suffered from a complicated non-violent RBD associated with sleep talking and simple movements of the extremities as revealed by video PSG (8). She was diagnosed with RBD because she exhibited RWA on PSG and had dream enactment-related problematic behaviors upon observation according to the criteria from the 2nd edition of the International Classification of Sleep Disorders (ICSD) (2). Her apnea-hypopnea index was $6.6 / \mathrm{h}$. Stridor is associated with decreased survival in patients with MSA, and often occurs in tandem with sleep apnea (9). Therefore, she was prescribed ramelteon $(8 \mathrm{mg} / \mathrm{day})$ due to the adverse effects of clonazepam and because she was predicted to suffer from a complicating sleep apnea syndrome in the future, due to the progression of her MSA. While on ramelteon, she benefited from a mild improvement in the occurrence of her RBD symptoms. Moreover, a second PSG showed a decreased percentage of RWA, from $8.5 \%$ to $3.5 \%$ of her sleep time. We checked her severity of RBD using the RBD severity scale (RBDSS) (10). Her RBDSS score improved from $(1,1)$ on the first examination with PSG to $(1,0)$ on the second. When she stopped taking ramelteon, her RBD symptoms reappeared. Following two years of treatment with ramelteon, we evaluated the severity of her RBD using the Japanese version of the RBD questionnaire (RBDQJP) $(11,12)$. Her scores on the RBDQ-JP improved from 41 in 2009 to 14 in 2012.

\section{Case 2}

A 58-year-old, right-handed man began suffering from a resting tremor of the right lower limb in 1993. At the same time, he was diagnosed with Parkinson's disease (PD) based on the established diagnostic criteria (13) and began treatment with dopaminergic agents in our department. He started to shout during his sleep in 1999 , and frequently experienced nocturnal visual hallucinations and dream enactment behavior during sleep. Although he took clonazepam for these symptoms, he continued to suffer from this dream enactment behavior. In 2010, he was admitted to our hospital for an adjustment of his treatments for both parkinsonian symptoms and nocturnal symptoms. Upon admission, he displayed a resting tremor of the left upper and lower limbs, a rigidity of the bilateral upper and lower limbs, a dropped neck, a forward-inclining posture as he walked, steppage gait and retropulsion. The severity of his parkinsonian symptoms was estimated to be Stage 3 on the Hoehn \& Yahr Scale, and at 65 points on the Unified Parkinson's Disease Rating Scale. Upon admission, he was taking levodopa (700 mg/day), entacapone (700 mg/day, five doses), selegiline $(2.5 \mathrm{mg} / \mathrm{day})$, droxydopa (200 $\mathrm{mg} / \mathrm{day}$, two doses), amantadine $(250 \mathrm{mg} / \mathrm{day}$, three doses) zonisamide $(100 \mathrm{mg} /$ day, one dose), ropinirole (6 mg/day, three doses) and clonazepam (1 mg/day, one dose). Cranial MRI found slightly chronic ischemic changes on the bilateral white matter by the lateral ventricle. SPECT showed a diffuse hypoperfusion 


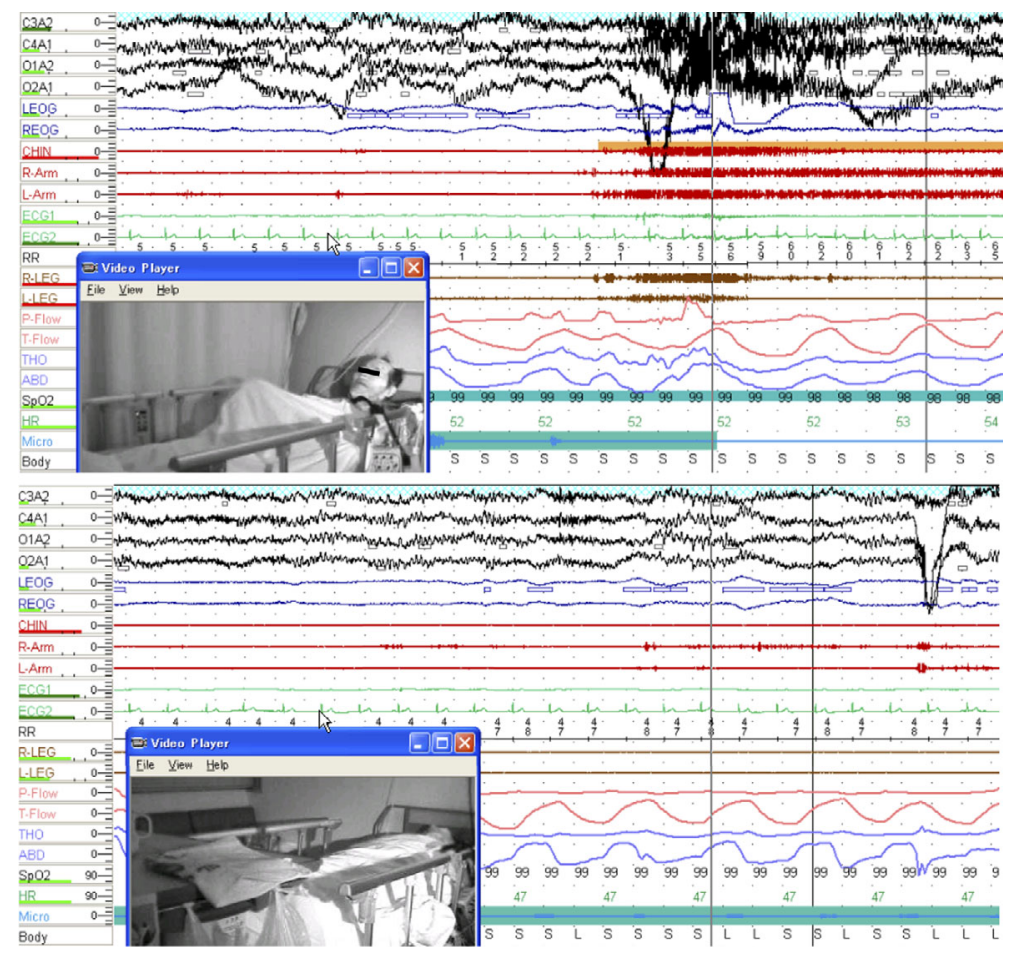

Figure. Video polysomnographies (PSG) before and after taking ramelteon. We examined the video PSG recordings from Case 2 that were taken before and after the initiation of the ramelteon treatment. Video PSG recordings included the following: electroencephalograpies with 4-channel scalp montages (C3, C4, O1, and $\mathrm{O} 2$ referred to the contralateral ear: $\mathrm{C3A2}, \mathrm{C} 4 \mathrm{A1}$, O1A2, and O2A1), electrooculographies (LEOG and REOG), electromyographies of the submental area and the bilateral upper and lower limbs (CHIN, R-Arm, L-Arm, R-LEG, and L-LEG), electrocardiographies (ECG1 and ECG2), respiratory rates (RR), oronasal airflow monitoring using both nasal pressure and thermistors (P-Flow and T-Flow), the measurements of the thoracic and abdominal respiratory movements with a strain gauge (THO and $\mathrm{ABO})$, transcutaneous oxygen saturation $\left(\mathrm{SpO}_{2}\right)$, heart rates (HR), microphones (Micro), positioning of body (Body) and video monitor (Video Player). Prior to the ramelteon therapy, he got up and shouted during REM sleep. He also reported being chased by strangers in his dream (upper video PSG). After taking ramelteon, he rarely reported dreaming. He displayed only simple movements of the chin and extremities during REM sleep (lower video PSG). Moreover, his phasic and tonic EMG in his chin during REM sleep decreased. As a result, the PSG findings revealed a dramatic reduction in the percentage of RWA per sleep time.

of the bilateral cerebella. MIBG myocardial scintigraphy indicated a reduced $\mathrm{H} / \mathrm{M}$ ratio on both early and delayed images (early image: 1.64, delayed image: 1.37). The results of the PSG recordings are detailed in Table. PSG revealed that he suffered from RWA symptoms during $10.9 \%$ of his sleep time. He got up and shouted during PSG video monitoring (Figure). He reported dreaming that strangers were chasing him. He was diagnosed with PD-complicated RBD according to the criteria from the 2nd edition of the ICSD (2). Although he was taking clonazepam at the time of the sleep study, this drug was not effective for his RBD. Once he began treatment with ramelteon $(8 \mathrm{mg} /$ day prior to sleeping), he rarely experienced dreams and had no RBD symptoms. During the ramelteon treatment course, a second PSG showed a decrease in the RWA symptoms from $10.9 \%$ to $3.9 \%$ of his sleep time. His RBDSS score improved from (3, $1)$ on the first examination using PSG to $(1,1)$ on the second. When we stopped prescribing ramelteon for him, he began to experience RBD symptoms again. As a result, he remains on ramelteon, and has now been taking it for three years. We determined that his RBDSQ-JP score improved from 70 in 2009 to 17 in 2012.

\section{Discussion}

Generally, clonazepam is a first-line treatment for RBD (2). However, clonazepam is not always effective for treating RBD symptoms. Clonazepam produces numerous side effects including dizziness, daytime sleepiness and muscle weakness. MSA is often complicated with SAS. Since the existence of SAS can be fatal in progressive states of MSA, we should avoid prescribing clonazepam for MSA patients with SAS.

Melatonin has been supported as a level B treatment for idiopathic RBD (3). Boeve et al. reported that melatonin was an effective treatment in 14 patients with secondary 
RBD (14). Their data support the notion that melatonin can be considered as a possible stand-alone or add-on therapy in select patients with RBD. In addition, several other medications, such as pramipexole, donepezil, Yi-Gan San and paroxetine, have been categorized as level $\mathrm{U}$ for the treatment of RBD in patients with PD (15). Ramelteon is one of the many melatonin receptor agonists available and produces few adverse effects (4). Ramelteon is thought to function similarly to melatonin. Patients with secondary RBD generally suffer from additional motor symptoms such as paralysis and parkinsonism. In such patients, careful attention should be paid to the risk of falls at nighttime. Melatonin and ramelteon may represent useful therapies in lessening the possibility of falling. Moreover, it was reported that treatment with melatonin protects L-DOPA from autoxidation in the striatum, and this class of medication might exhibit neuroprotective effects (16). RBD is often complicated with neurodegenerative diseases, and RBD might also represent prodromal symptoms of several neurodegenerative diseases (17). If ramelteon exhibits neuroprotective effects, it might be beneficial in patients with idiopathic RBD in the prevention of neurodegenerative disease states.

In conclusion, we evaluated the effects of ramelteon for RBD via subjective and objective evaluations in two patients with secondary RBD, and our findings indicate the possible efficacy of ramelteon for the treatment of RBD. Ramelteon might also be useful for patients with RBD who are unable to take clonazepam and/or are resistant to clonazepam treatment. In order to verify these findings, it will be necessary to construct double-blinded placebo trials, including additional patients with idiopathic and secondary RBD, along with long-term, follow-up studies employing both subjective and objective measurements.

The authors state that they have no Conflict of Interest (COI).

\section{References}

1. Schenck CH, Bundlie SR, Mahowald MW. Delayed emergence of a parkinsonian disorder in $38 \%$ of 29 elder men initially diagnosed with idiopathic rapid eye movement sleep behavior disorder. Neurology 46: 388-393, 1996.

2. American Academy of Sleep Medicine. International Classification of Sleep Disorders: Diagnostic and Coding Manual. 2nd ed. Westchester, IL, 2005: 148-152.

3. Aurora RN, Zak RS, Maganti R, et al. Best practice guide for the treatment of REM sleep behavior disorder (RBD). J Clin Sleep Med 6: 85-95, 2010.

4. Uchiyama M, Hamamura M, Kuwano T, Nishiyama H, Nagata H, Uchimura N. Evaluation of subjective efficacy and safety of ramelteon in Japanese subjects with chronic insomnia. Sleep Med 12: 119-126, 2011.

5. Rechtschaffen A, Kales A. A Manual of Standardized Terminology, Techniques and Scoring System for Sleep Stages in Human Subjects. U.S. Government Printing Office, National Institute of Health Publication, Washington DC, 1968.

6. American Sleep Disorders Association. Manuals of the Scoring of Sleep and Associated Events. Rules, Technology, and Technical Specifications. 2007: 42-43.

7. Gilman S, Low P, Quinn N, et al. Consensus statement on the diagnosis of multiple system atrophy. American Autonomic Society and American Academy of Neurology. Clin Auton Res 8: 359362, 1998.

8. Nomura T, Inoue Y, Högl B, et al. Comparison of the clinical features of rapid eye movement sleep behavior disorder in patients with Parkinson's disease and multiple system atrophy. Psychiatry Clin Neurosci 65: 264-271, 2011.

9. Ghorayeb I, Bioulae B, Tison F. Sleep disorders in multiple system atrophy. J Neural Transm 112: 1669-1675, 2005.

10. Sixel-Doring F, Schweitzer M, Mollenhauer B, Trenkwalder C. Intraindividual variability of REM sleep behavior disorder in Parkinson's disease: a comparative assessment using a new REM sleep behavior disorder severity scale (RBDSS) for clinical routine. J Clin Sleep Med 15: 75-80, 2011.

11. Li SX, Wing YK, Lam SP, et al. Validation of a new REM sleep behavior disorder questionnaire (RBD-HK). Sleep Med 11: 43-48, 2010.

12. Sasai T, Matsuura M, Wing YK, Inoue Y. Validation of the Japanese version of the REM sleep behavior disorder questionnaire (RBDQ-JP). Sleep Med 13: 913-918, 2012.

13. Daniel SE, Lees AJ. Parkinson's Disease Society Brain Bank, London: overview and research. J Neural Transm 39 (Suppl): S 165-S172, 1993.

14. Boeve BF, Silber MH, Ferman TJ. Melatonin for treatment of REM sleep behavior disorder in neurologic disorders: results in 14 patients. Sleep Med 4: 281-284, 2003.

15. Zesiewicz TA, Sullivan KL, Arnulf I, et al. Practice of parameter: treatment of nonmotor symptoms of Parkinson disease: report of the quality standards subcommittee of the American Academy of Neurology. Neurology 74: 924-931, 2010.

16. Rocchitta G, Migheli R, Esposito G, et al. Endogenous melatonin protects L-DOPA from autoxidation in the striatal extracellular component of the freely moving rat: potential implication for long-term L-DOPA therapy in Parkinson's disease. J Pineal Res 40: 204-213, 2006.

17. McCarter SJ, Louis EK, Boeve BF. REM sleep behavior disorder and REM sleep without atonia as an early manifestation of degenerative neurological disease. Curr Neurol Neurosci Rep 12: 182192, 2012.

(C) 2013 The Japanese Society of Internal Medicine http://www.naika.or.jp/imonline/index.html 